\title{
Voz y Acción en el Instituto: Cómo el Alumnado de Secundaria Percibe su Participación
}

\author{
Voice and Action in School: How Secondary School Students \\ Perceive their Participation
}

\author{
Laura Granizo 1* \\ Kevin van der Meulen ${ }^{2}$ \\ Cristina del Barrio ${ }^{2}$ \\ ${ }^{1}$ Universidad a Distancia de Madrid, España \\ ${ }^{2}$ Universidad Autónoma de Madrid, España
}

\begin{abstract}
La participación es uno de los ejes de la justicia social y de la inclusión dado que involucra uno de los derechos de la infancia y la adolescencia. Se realizó un estudio ex post facto prospectivo con el objetivo de comprender el significado que tienen los adolescentes del concepto de participación y de sus prácticas. Fueron un entrevistados un total de 71 estudiantes voluntarios (entre 13 y 17 años; 63\% chicas) de tres centros públicos de secundaria de la Comunidad de Madrid. La mayor parte del alumnado relaciona la participación con un proceso voluntario y que refleja la necesidad de dar su opinión ante los temas que les afectan. También se apoya la idea de buscar el bienestar grupal como objetivo de la participación. Además, los estudiantes consideran la participación como necesaria para el buen funcionamiento del centro, si bien, en la realidad consideran tener una participación reactiva ante las propuestas del profesorado. Las concepciones de los estudiantes son coherentes con el modelo de justicia social e inclusión por el que se garantiza el derecho del alumnado a la participación en nuestro sistema educativo, sobre todo entre las chicas. No obstante, parece que su participación es poco proactiva quedando su rol de agentes difuminado.
\end{abstract}

Descriptores: Participación estudiantil; Justicia social; Educación ciudadana; Enseñanza secundaria; Entrevista.

Participation is one of the core areas of social justice and inclusion as it involves one of the children and adolescents' rights. A prospective ex-post-facto study was carried out aiming at identifying the practices and meaning of participation among adolescents. A total of 71 volunteer students (13-17 years of age; $63 \%$ girls) from three state-funded secondary schools in the Community of Madrid were interviewed. Most students associate participation with a voluntary process, which reflects their need for giving their opinions on issues that concern them. The idea of searching for the groups' wellbeing as an aim of participation is supported by the students. Moreover, they see participation as necessary for the school's well-functioning, although they evaluate their participation as reactive, as compliance with the teachers' proposals. Students' conceptions are consistent with a model of social justice and inclusion in which students' right to participation is guaranteed within the educational system, mostly among girls. However, students do not seem to be proactive in their participation pointing at a timid exercise of their role as agents.

Keywords: Student participation; Social justice; Citizenship education; Secondary education; Interviews.

*Contacto: laura.granizo@udima.es

ISSN: 2254-3139

www.rinace.net/riejs/

revistas.uam.es/riejs
Recibido: $\quad 11$ de septiembre 2019

$1^{\text {a }}$ Evaluación: 7 de noviembre 2019

$2^{\text {a }}$ Evaluación: 9 de diciembre 2019

Aceptado: $\quad 14$ de diciembre 2019 


\section{Revisión de la literatura}

Hablar de justicia social en educación implica intentar promover con las prácticas educativas el bienestar y el pleno desarrollo de todas las personas beneficiarias de las mismas. Sin embargo, son múltiples las barreras a las que se enfrenta esta manera inclusiva de entender la educación. Hay numerosos estudios, y no por ello hay que dejar de prestarle atención, acerca de los procesos de exclusión a los que se enfrentan colectivos especialmente vulnerables por sus condiciones personales o sociales (Del Barrio. Martín, Almeida y Barrios, 2003; Echeita, 2008; Granizo, Naylor y del Barrio, 2006; Slee, 2010; Unesco, 2001). Pero, además, en los centros existen barreras para la inclusión que afectan al conjunto del alumnado, como son las prácticas educativas en que no se sienten partícipes (Connell, 1993; Flutter, 2006; Santos Guerra, 2007), y que en parte tienen su origen en las concepciones del profesorado (Biesta, 2016).

La inclusión en general, y en el ámbito educativo en particular, se basa en tres ejes centrales (Echeita, 2014), a saber: presencia (acceso y permanencia de todo el alumnado en los mismos centros), rendimiento (posibilitar el máximo desarrollo de todas las capacidades de cada estudiante) y participación (ser y sentirse parte de los centros escolares). También si hablamos de justicia social nos referimos a tres dimensiones: redistribución de recursos, reconocimiento y representación (Fraser, 2008). Si bien hay mucho margen de mejora en los tres parámetros que sustentan los modelos inclusivos y de justicia social, hay uno especialmente preocupante cuando ponemos el foco en el alumnado: el de la participación o representación (Cochran-Smith, 2010). La participación del alumnado es uno de los objetivos centrales de los sistemas educativos que pretenden contribuir al desarrollo integral de los estudiantes para capacitarles a vivir en democracia. Así fue recogido por la unesco (1996) cuando señaló este aspecto como uno de los pilares de la educación del siglo xxi, y así ha quedado también plasmado en nuestras leyes educativas. Además, es un derecho de la infancia y la adolescencia y como tal debe ser garantizado por los adultos y la sociedad en su conjunto. Los niños, niñas y adolescentes deben tener agencia, es decir, la oportunidad de opinar y actuar en los temas que les afectan directamente (Hart, 1992; Rudduck y Flutter, 2003; Save the Children, 2005) dejando de considerarse de manera pasiva y como meros receptores de ayuda (Nussbaum, 2012). Pero es importante que esta participación gire en torno a aspectos centrales de la vida escolar, como el currículo, la metodología o la gestión, y que no se limite a aspectos periféricos (Kim, 2015). Su voz es central para que los procesos de enseñanza y aprendizaje en las aulas sean eficaces (Mitra, 2018; Simmons, Graham y Thomas, 2015). Además, la participación real de los estudiantes en los centros tiene un alineamiento claro con la necesidad de trabajar por competencias, es decir, buscando el carácter práctico y la transferencia de lo aprendido a la realidad compleja en la que vivimos (Zabala y Arnau, 2007). Y es un requisito básico para enseñar qué implica la democracia tanto desde el punto de vista actitudinal como procedimental y conceptual (Print, 2007).

No existe una definición única del concepto de participación referida al contexto escolar (Ceballos-López y Saiz-Linares, 2019). Para algunos autores, por ejemplo, supone pensar en aprender con otros y colaborar con ellos, implicarse y ser reconocido y aceptado por lo que uno es (Booth, 2006). No obstante, los autores parecen estar de acuerdo en la existencia de un continuo construido en base a la involucración del alumnado, estando en un extremo las modalidades más pasivas o incluso simbólicas y en el otro las que implican un ejercicio compartido del poder (Bisquerra, 2008; Hart, 1992; Susinos-Rada y Ceballos- 
López, 2012). En este trabajo hemos tomado como referencia la propuesta de BlackHawkins, Florian y Rouse (2007) que hablan de tres tipos de participación equivalentes a los tres ejes de la educación inclusiva que mencionábamos anteriormente. Así, construyen su Marco de Referencia para la Participación de la siguiente manera: a) participación y acceso: estar ahí; b) participación y colaboración: aprender juntos; y c) participación y diversidad: reconocimiento y aceptación.

A pesar de que hay estudios que apuntan a una baja participación de los estudiantes en la escuela (Coiduras et al., 2016; Martínez y Aróstegui, 2001) existen prácticas bien documentadas de centros que dan voz a los estudiantes en la gestión de los mismos y de los beneficios asociados (Cook-Sather, 2014). Unos se sustentan en el cuidado del clima moral del centro, como los sistemas de ayuda entre iguales (Cowie y Wallace, 2000), y otros en aspectos más organizativos, como la elaboración de las normas y la toma de decisiones, como en el programa de las Just Community Schools (Power, Higgings y Kohlberg, 1989), si bien este último solo ha sido puesto en práctica en centros de tamaño pequeño. También la iniciativa triple $\mathrm{R}$ (rights, respect, responsability) llevada a cabo en Inglaterra, de cuya aplicación se concluye que una manera eficaz de ayudar a los adolescentes a ser ciudadanos respetuosos es a través de prácticas que aúnan la participación con el conocimiento de los propios derechos (Covell, Howe y McNeil, 2008). Los estudios de Quinn y Owen (2016), por su parte, analizan el impacto de la participación institucional del alumnado a través de los consejos estudiantiles. En la etapa de Educación Primaria destacan algunos centros como los analizados por García-Pérez y Montero (2014) que, a través del trabajo asambleario del alumnado y las familias, posibilitando su participación en la toma de decisiones, han conseguido mejorar las actitudes del alumnado hacia la escuela, las relaciones interpersonales entre los estudiantes y también con el profesorado y, en definitiva, crear un sentido de comunidad y de pertenencia. También en la etapa de Educación Infantil dar voz al alumnado y a sus familias ha mostrado efectos positivos en la organización del espacio escolar (Clark, 2010; Ceballos-López, SusinosRada y García-Lastra, 2018). Favorecer la participación del alumnado y darles voz en aquellos temas que les afectan directamente no solo es un derecho, sino además una manera de mejorar el clima escolar (Edwards y Mullis, 2003), el bienestar del alumnado (Powell et al., 2018), la creatividad de los alumnos (Serrano, Soto, Arcos y Jarquín, 2018) y su preparación para el compromiso cívico futuro (Flanagan et al., 1998; Haste, 2005; Rodríguez, Kohen, Delval y Messina, 2018). Además, favorece la construcción de una cultura más democrática entre el profesorado (Saiz-Linares, Ceballos-López y SusinosRada, 2019). También se ha mostrado como una manera eficaz de posibilitar aprendizajes recogidos en las programaciones docentes mientras se da respuesta a alguna necesidad del entorno social, gracias a las experiencias de Aprendizaje Servicio (Traver-Martí, Moliner y Sales, 2019).

A pesar de que existen algunos estudios centrados en el análisis de la participación del alumnado, hay un vacío respecto a su concepción acerca de este proceso, si bien el estudio de las representaciones de las personas es una de las líneas de la justicia social en educación (Murillo y Hernández-Castilla, 2011). ¿Qué se representan los alumnos y alumnas cuando escuchan o cuando deben describir este concepto? Desde nuestra posición teórica entendemos que la participación de los estudiantes es un elemento imprescindible para conseguir el desarrollo integral de los estudiantes y para lograr un clima o cultura escolar preocupado por el bienestar colectivo. Pero, ¿piensan lo mismo los estudiantes? ¿Qué valoración hacen de su participación? ¿Cuáles son sus experiencias participativas? 
Intentaremos responder a estas cuestiones a través de los siguientes objetivos específicos: 1) analizar la concepción del alumnado acerca del proceso de participación en los centros; 2) conocer su experiencia participativa dentro de los centros.

\section{Método}

Para dar respuesta a los objetivos planteados se realizó un estudio ex post facto prospectivo (Montero y León, 2007). El estudio que aquí se presenta forma parte de un proyecto más amplio que constituyó la tesis doctoral de la primera autora centrado en las ideas y experiencias de participación dentro del centro escolar y en su comunidad, comparando grupos de estudiantes con el rol de delegados y/o compañeros ayudantes y grupos sin ese papel para ver posibles diferencias en su concepción de la participación a partir de su propia experiencia como agentes de inclusión educativa (Granizo, 2011).

\section{Participantes}

Participaron 71 estudiantes ( 45 chicas y 26 chicos) con edades comprendidas entre los 14;5 (años; meses) y los 17; 5 (años; meses) (con una media de edad de 15;9) pertenecientes a tres centros públicos de la Comunidad de Madrid. Los centros fueron escogidos por muestreo incidental. Nos pusimos en contacto con los centros a través de sus Departamentos de Orientación. Estos a su vez se pusieron en contacto con los tutores de los grupos quienes se encargaron de informar a las familias a través de una circular. Todos los adolescentes de los centros implicados fueron autorizados a participar por sus familias, aunque solo lo hicieron los que, tras haber explicado en sus clases los objetivos de la investigación, se ofrecieron de manera voluntaria.

\section{Instrumento}

Los estudiantes fueron entrevistados de manera individual siguiendo el método clínicocrítico piagetiano (Delval, 2001), es decir, partiendo de una serie de preguntas sobre los aspectos objeto de estudio, a modo de guion, pero añadiendo nuevas cuestiones para profundizar a partir de lo que la persona entrevistada expresa. Las preguntas se agrupaban en los siguientes tres bloques de contenido: a) el objetivo de la educación y su relación con el concepto de ciudadanía, para ver si espontáneamente contemplan la participación como un posible objetivo educativo; b) la concepción de la participación de los estudiantes dentro de los centros: que estimen la magnitud de la participación del alumnado en el centro o en la clase, que analicen quién promueve esa participación y que señalen el valor que tiene para ellos mismos esa participación; c) las experiencias de participación real de cada estudiante entrevistado tanto dentro como fuera del centro: su grado de participación, la naturaleza de la misma y la comparación de ambos contextos.

La entrevista fue pilotada con 20 alumnos ( 13 chicas y 7 chicos) pertenecientes a una clase de $2^{\circ}$ de eso. Tras su valoración positiva en cuanto a contenido y extensión no fue necesario hacer cambios en las preguntas.

\section{Procedimiento}

Los estudiantes eran entrevistados individualmente en alguna sala tranquila del propio centro cedida por la jefatura de estudios. Tras una breve presentación personal, el entrevistador/a introducía el objetivo del estudio resaltando que se quería conocer la opinión de los adolescentes acerca de cómo era su vida en los centros y que, por tanto, todas las respuestas eran igualmente valiosas y correctas de cara a la investigación. 
Además, se recordaba que las respuestas que se dieran individualmente solo serían conocidas por los investigadores.

La entrevista era grabada en audio con la autorización de la persona entrevistada y transcrita literalmente para poder ser analizada posteriormente con mayor objetividad. Las respuestas se analizaron cualitativamente por medio de la elaboración de categorías o niveles de respuesta que recogieran las semejanzas y diferencias en el pensamiento expresado por los participantes. En este proceso se utilizó programa QDA-Miner. Las categorías fueron validadas a través de un análisis interjueces del $20 \%$ de las entrevistas (14 del total de 71) escogidas al azar. Los porcentajes de acuerdo entre jueces oscilaron entre el $80 \%$ y el $100 \%$ dependiendo del bloque de contenido.

A continuación, se analizó de manera cuantitativa la distribución porcentual de las respuestas del total de los participantes en dichas categorías, así como la distribución por género. Al tratarse de variables categóricas, para comprobar la significación estadística de las diferencias se usaron las pruebas estadísticas de $\chi^{2}$ utilizando el paquete estadístico spss (Field, 2005).

\section{Resultados}

\subsection{La participación como objetivo educativo}

Cuando los estudiantes hablaban del objetivo que consideraban que tenía la escuela, además de atribuirle un carácter propedéutico, la mayoría aludía al desarrollo personal del alumnado, pero desde un punto de vista individual más que social. Cuando se les preguntaba a los estudiantes directamente si creían que formar buenos ciudadanos era uno de los objetivos de la escuela encontrábamos que el 94,3\% estaba de acuerdo. No obstante, cuando se les preguntaba por la relación entre ser un buen ciudadano y participar, ya que vivimos en una sociedad democrática, solo un tercio del alumnado consideraba que era necesario participar, y casi el doble lo valoraba como algo positivo, aunque no necesario (figura 1). El resto no consideraba que existiera relación entre ambos conceptos.

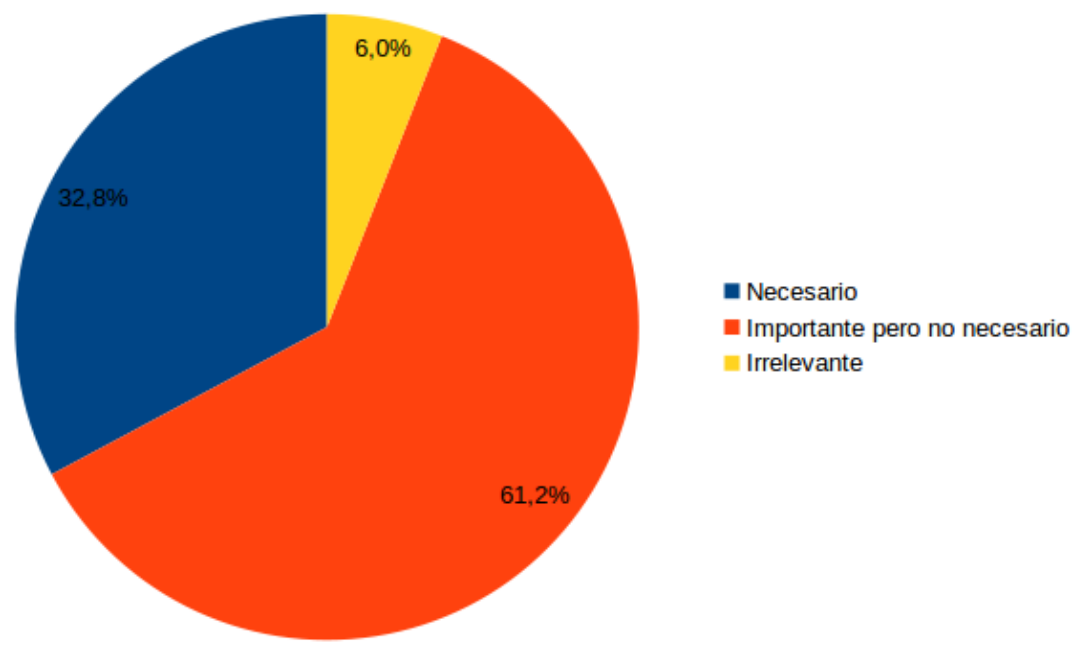

Figura 1. Distribución de la relevancia de la participación en la representación de un buen ciudadano $(\%)(\mathrm{N}=67)$

Fuente: Recuperado de Granizo (2011, p. 235). 


\subsection{El concepto de participación}

Como ya hemos señalado el concepto de participación no activa el mismo esquema mental en todas las personas. Por eso, se preguntó a los estudiantes qué entendían ellos por participar o cómo se podía participar en sus institutos. A partir de las respuestas obtenidas se construyeron categorías temáticas (cuadro 1) que construyen un continuo congruente con la propuesta de Black-Hawins, Florian y Rouse (2007), cuyas categorías se relacionarán con las construidas en este estudio. Las respuestas de los estudiantes en esta pregunta podían encajar en más de una categoría atendiendo a los aspectos de contenido a los que hicieran referencia.

Cuadro 1. Ejemplos de los elementos constitutivos del concepto de participación según el alumnado $(\mathrm{N}=71)$

\begin{tabular}{|c|c|c|}
\hline & $\begin{array}{ll}\text { EJEMPLOS } \\
\end{array}$ & $\%$ \\
\hline $\begin{array}{l}\text { Acatar y } \\
\text { respetar }\end{array}$ & $\begin{array}{l}\text { “[¿Qué sería para ti participar?] Pues hacer lo que hace la mayoría” } \\
\text { (Chico, 15;6) } \\
\text { "Participar es, yo qué sé, aceptar cualquier cosa que te den, si te dicen } \\
\text { cualquier cosa pues tú aceptarla sea del tipo que sea mientras } \\
\text { tampoco perjudique” (Chico, } 15 ; 2 \text { ) }\end{array}$ & 18,3 \\
\hline Relacionarse & $\begin{array}{l}\text { "Entiendo participar no sólo como la parte democrática sino también } \\
\text { un poco como interactuar con las personas de tu medio, de tu } \\
\text { ciudad, tu país" (Chica, 14;10) } \\
\text { “(Participar es) incluirte en alguna actividad o algo, en lo que sea. } \\
\text { Participar en un proyecto de trabajo, yo qué sé, en algún } \\
\text { concurso"(Chica, 14;7) }\end{array}$ & 25,4 \\
\hline Opinar & $\begin{array}{l}\text { "Participar es hablar en grupo, hablar en sociedad, elegir una opción, } \\
\text { dar opciones, eso es una participación (...) Es importante aportar } \\
\text { algo, te lo acepten o no, para defender tus ideas. Si tú piensas que la } \\
\text { clase va ir a mejor con otra norma, pues defiende tus ideas y dilo. Si } \\
\text { no defiendes las ideas que tú tienes, no haces nada" (Chico, 16;5) }\end{array}$ & 52,1 \\
\hline $\begin{array}{l}\text { Buscar el } \\
\text { bienestar } \\
\text { grupal }\end{array}$ & $\begin{array}{l}\text { “[¿Qué sería para ti participar?]En participar en algo para la ciudad, } \\
\text { para todos. No sé, como cuando participas en lo de reciclar y eso. } \\
\text { [¿Por qué eso es participar?]Porque pones de tu parte” (Chica, 16;7) } \\
\text { "Yo creo que participar es pensando más en el resto de la clase, en } \\
\text { toda la clase, no en yo y mi nota" (Chica, 15;5) } \\
\text { "Cuando hay campañas de limpieza, o cuando puedes donar sangre... } \\
\text { supongo que colaborando a que lo que te rodea esté bien. Pagando } \\
\text { unos impuestos que ayuden a mantener todo" (Chica, 14;9) }\end{array}$ & 42,3 \\
\hline Voluntario & $\begin{array}{l}\text { "Participar es hacer algo que tú quieres" (Chico, 15,5) } \\
\text { "Pues sí, las normas hay que respetarlas, que para eso están, pero no lo } \\
\text { considero una forma de participar porque no estás dando tu opinión, } \\
\text { sólo estás haciendo lo que ellos te obligan a hacer. [Entonces, } \\
\text { ¿participar es algo que tiene que salir de ti?] Claro, que no te } \\
\text { obliguen" (Chica, 14;8) }\end{array}$ & 12,7 \\
\hline
\end{tabular}

Fuente: Recuperado de Granizo (2011, p. 237-238).

En el extremo inferior estaban las respuestas menos maduras (entendiendo la madurez desde un punto de vista de desarrollo moral de preocupación por el bienestar propio y ajeno y de agencia). Así, encontrábamos una concepción pasiva de la participación: acatar y respetar. Según los alumnos que indicaban este aspecto, participar suponía asumir lo que hace la mayoría sin poner trabas a aquello que quieren los demás y sin significarse. Es decir, participar sería equivalente a dejar que todo siga su curso. Equivaldría a la participación como acceso o "estar ahí". Para otros, sin embargo, la participación suponía relacionarse con otros. Se asociaría con la categoría de "participación y colaboración”. En esta categoría el peso estaba en establecer relaciones con otras personas, compartir 
tiempos, espacios y actividades con los demás. No se concretaba aquí quién tenía la iniciativa ni cómo se decidían las actividades.

Por otro lado, hay quienes consideraban que participar suponía opinar. Para estos solo se podía hablar de participación cuando todos los implicados comparten su punto de vista con los demás acerca de lo que se está haciendo. Señalaban que hay distintas maneras de expresarse, siendo unas más convencionales y estando más reguladas que otras, por ejemplo, votar, escribir quejas o reclamaciones ciudadanas, etc. Otras tendrían un carácter más informal, como participar en un debate. En cualquiera de los casos, se entendería la participación como una manera de posicionarse de manera explícita ante los demás y, por tanto, supondría una inversión personal. Equivaldría a la participación y diversidad o "reconocimiento y aceptación".

Y, por último, en el extremo superior del continuo, el que entenderíamos como más maduro desde un punto de vista moral, estarían los que asociaban la participación con la búsqueda del bienestar grupal o con la mejora de la comunidad. Cuando se hace algo solo movido por el interés particular no podríamos hablar de participación. Además, para algunos adolescentes solo se podría hablar de participación cuando la persona decidiera de manera libre y voluntaria llevar a cabo una determinada acción. De ser impuesta o forzada, tampoco podríamos hablar de este proceso.

Una de las ventajas del método clínico-crítico piagetiano usado en este trabajo es que permite indagar sobre los objetivos de estudio no solo a través de las preguntas directamente relacionadas con los mismos sino también a partir de las respuestas dadas en distintos momentos de las entrevistas. Así, aunque se pedía a los estudiantes definir el concepto de participación mediante una pregunta directa, sus concepciones también surgían de manera espontánea en otros momentos de la entrevista, por ejemplo, al valorar conductas como preguntar en clase, elaborar las normas o elegir al delegado. Por eso, en esta pregunta nos pareció interesante diferenciar ambos aspectos. Llamaremos directa a las respuestas explícitas a la pregunta y total a las deducidas del conjunto de la entrevista. Si comparamos ambos tipos de respuesta podemos encontrar (figura 2) que si bien todas las características de la participación se veían reforzadas había una que crecía de manera destacada, el carácter voluntario, que pasaba a ser mencionado del $12,7 \%$ al $46,5 \%$.

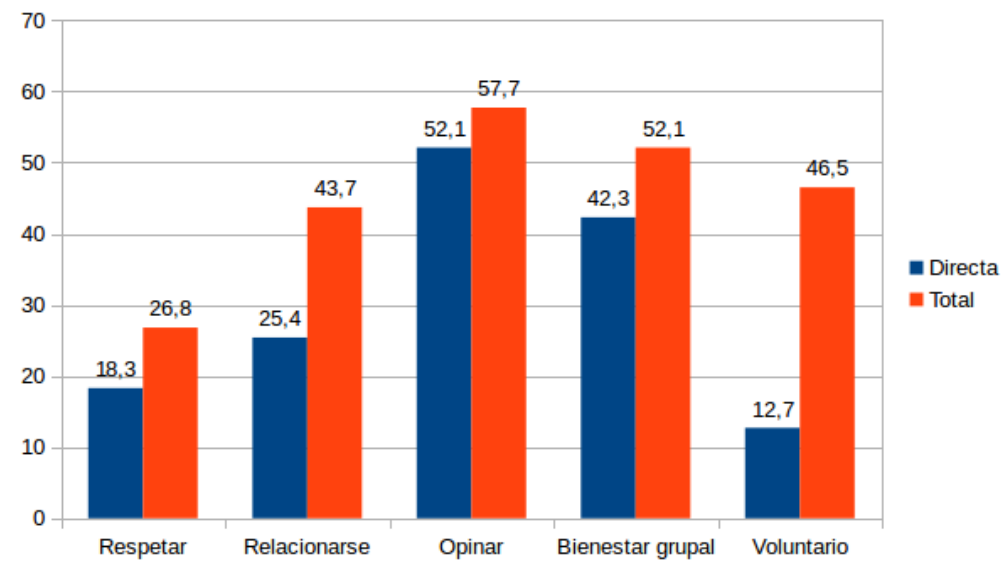

Figura 2. Elementos del concepto de participación señalados por el alumnado al ser preguntados de forma directa o a lo largo de la totalidad de la entrevista $(\% ; \mathrm{N}=71$ )

Fuente: Recuperado de Granizo (2011, p. 239). 
Esto nos hizo pensar que se trataba de una concepción más implícita del término pero que resultaba inherente al mismo para muchos estudiantes. Además, la proporción de chicas que señalaban que la participación debería ser voluntaria $(55,6 \%)$ era mayor que la de $\operatorname{chicos}(30,8 \%)\left(\chi^{2}(1)=4,070 \mathrm{p}=0,044\right)$.

\subsection{La naturaleza y el grado de participación}

Una vez conocida su representación de qué es participar, queríamos conocer su valoración acerca de la participación en sus institutos. En primer lugar, se les preguntaba por la importancia que otorgaban a participar en el centro. A diferencia de la relación que establecían entre ciudadanía y participación, en este caso la mayoría considera que la participación del alumnado era necesaria para que el buen funcionamiento de la institución $(66,7 \%)$. Esto era defendido por el $78 \%$ de las chicas y por el $48 \%$ de los chicos $\left(\chi_{(1)}^{2}=6,3\right.$ $\mathrm{p}=0,012)$. Un $27,3 \%$ consideraba que no era obligatorio, pero sí era positivo, y tan solo un $6 \%$ la consideraba irrelevante.

$\mathrm{Al}$ analizar antes el concepto de participación nos referíamos a que el carácter voluntario de la misma parecía teñir las concepciones de la mayoría del alumnado. ¿Pero cuál es la naturaleza de la participación? ¿Quién tiene la iniciativa de las actividades llevadas a cabo? La distribución del poder en el centro puede encontrar también una manifestación en las

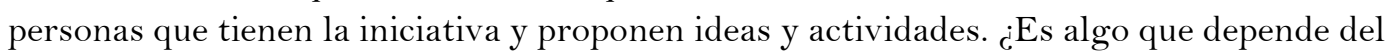
profesorado, del alumnado o es una decisión compartida? Teniendo esto en mente la mayor parte del alumnado $(52,4 \%)$ consideraba que los profesores eran los promotores de las ideas y las propuestas. El papel de los chicos y chicas era reactivo a pesar de que sí podían decidir si participar o no. Consideraban, por ejemplo, que "siempre que nos dice la profesora alguna actividad, la acabamos haciendo, pero no solemos decir nosotros ninguna idea ni nada" (Chico, 15;2), o que "lo de que los alumnos propongan una actividad, yo creo que podría estar bien, que lo hablemos en clase, en una excursión o algo, y que ya lo organicé el profesor. Estaría bien, pero no lo hacemos" (Chico, 15;0). En el otro extremo encontrábamos que son los estudiantes los que proponían y promovían distintas actividades en las que implicarse y participar, mostrando un carácter proactivo. Esta concepción solo la tenía el 3,2\% del alumnado entrevistado. Por último, habría un grupo de estudiantes que consideraba que la iniciativa era compartida entre profesorado y alumnado. Así, el 44,4\% del alumnado consideraban que la iniciativa se repartía y que "nosotros proponemos cosas y ellos las aceptan y ellos proponen cosas y nosotros las aceptamos" (Chica, 16;4)

Conocidas las características de la participación, la iniciativa y la importancia atribuida queríamos saber cómo estimaban ellos la participación de sus compañeros en el centro y en su clase. Clasificábamos la participación del alumnado como alta si consideraban que más de un $70 \%$ de los estudiantes participaba. Cuando lo hacía entre el 30 y el $70 \%$ hablábamos de participación media y, por último, considerábamos que era baja cuando afirmaban que participaba menos del $30 \%$ de los chicos y chicas. En la Figura 3 se puede observar la distribución de las tres opciones. Por género, los chicos estimaban la participación como alta en mayor proporción que las chicas $\left(\chi_{(1)}^{2}=4,9 \mathrm{p}=0,028\right)$. 


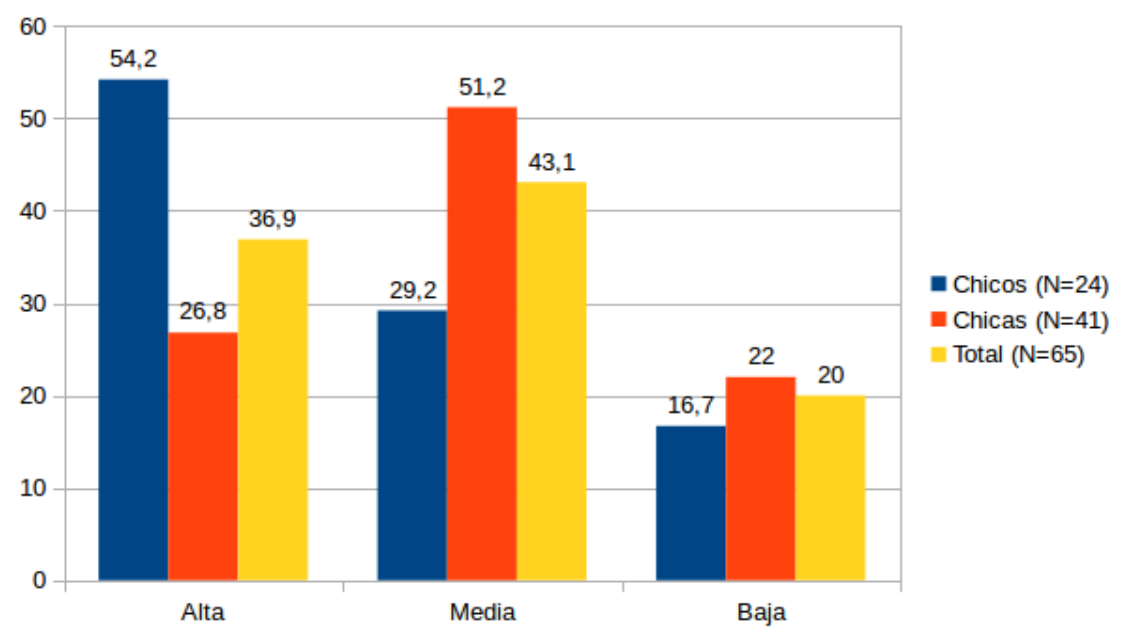

Figura 3. Distribución de la estimación de la participación del alumnado por género (\%) Fuente: Recuperado de Granizo (2011, p. 244).

\section{Discusión y conclusiones}

La participación del alumnado es uno de los pilares tanto de la educación inclusiva como de la justicia social referida al ámbito educativo. Cuando se analiza el objetivo número 4 de los Objetivos para el Desarrollo Sostenible de Naciones Unidas para 2030, nos encontramos con la necesidad de gestionar las culturas, las políticas y las prácticas educativas que posibiliten la calidad y la equidad en educación y que reduzcan las tasas de fracaso y abandono escolar, de manera que mejore la inclusión y la justicia social. Desde ambos enfoques el fomento de la participación del alumnado, y de la comunidad educativa en general, es uno de los objetivos que hay que lograr. Sin embargo, existen barreras en nuestro sistema educativo para gestionar los espacios y tiempos que posibiliten la misma, lo que lleva a hablar de un juego democrático trucado (Santos Guerra, 2007), porque las posibilidades de participación del alumnado se reducen a asuntos insustanciales y porque las decisiones en los centros se toman en foros jerárquicos, lo cual va en contra del espíritu de la inclusión (Flutter, 2006; Kim, 2015).

La participación del alumnado es clave para conseguir aprendizajes significativos y competenciales que se puedan transferir a la realidad. Las metodologías activas como el Aprendizaje Basado en Proyectos (García-Valcárcel y Basilotta, 2017) o las metodologías cooperativas (Pujolás, 2012), que dan voz al alumnado en su proceso de aprendizaje, muestran mejoras no solo en los aprendizajes académicos alcanzados sino también en la motivación y autoestima de los estudiantes y en el clima social de las clases (Powell et al., 2018). También las experiencias de Aprendizaje-Servicio, en las que pueden lograrse aprendizajes a través de la respuesta del alumnado a una necesidad social detectada, han concluido que la satisfacción del alumnado y su implicación con las prácticas realizadas se benefician de la responsabilidad y acción real que se les proporciona este tipo de formación (Uruñuela, 2018). A pesar de que los estudiantes son los principales implicados y de las múltiples prácticas y experiencias llevadas a cabo para mejorar la participación del alumnado, no existen apenas estudios que valoren el impacto de estas dinámicas en sus representaciones. Este estudio pretendía avanzar en esa línea a través de un doble objetivo: a) analizar la concepción del alumnado acerca del proceso de participación en los centros;

b) conocer su experiencia participativa en los mismos. 
Respecto al primer objetivo, partíamos de la relación entre educación y ciudadanía y entre ciudadanía y participación y analizábamos después el concepto de participación y escuela. La mayoría del alumnado consideraba que el objetivo de la escuela tenía que ver con formar buenos ciudadanos, aunque pensaban en un desarrollo más individual que social (Granizo, 2019). Además, creían que la participación era importante al pensar en un buen ciudadano, pero no era necesaria. Sin embargo, al pensar en el contexto escolar, la mayoría consideraba esencial la participación del alumnado para el buen funcionamiento del instituto. Por tanto, pensada de una manera más concreta y en un contexto más cercano, parece que los y las adolescentes valoran más su papel en el bienestar propio y en el de su entorno. Al profundizar en el significado de participación los adolescentes muestran ideas que se asemejarían al continúo construido por otros autores a partir de la cesión de responsabilidad en la toma de decisiones. En un extremo se refieren a lo que los autores han llamado "estar ahí" (Black-Hawkins, Florian y Rouse, 2007), "formar parte" (Bisquerra, 2008) o "participación simbólica" (Hart, 1992) y en el otro extremo se hablaría de "reconocimiento y participación" (Black-Hawkins, Florian y Rouse, 2007), "tener parte" (Bisquerra, 2008) o de acciones "iniciadas por niños y compartidas por los adultos" (Hart, 1992). Las respuestas más frecuentes de los entrevistados hablan de una concepción de la participación a medio camino del continúo expuesto. Así, más de la mitad del alumnado aludía a la necesidad de dar su opinión. Esto podría también entenderse como un reflejo de su comprensión del derecho de autodeterminación (López, 2008). Además, se aludía a la necesidad de buscar el bienestar grupal, entendiéndose la participación como una conducta de carácter prosocial (Hart, Atkins y Donally, 2006). En este sentido, los programas de alumnos ayudantes serían coherentes con este concepto de participación referida a un papel de los estudiantes como agentes, y explicarían los beneficios que los alumnos y alumnas encuentran en su implementación en los centros (Cowie et al., 2002; Del Barrio et al., 2011). La opción menos señalada por el alumnado sería la que representaría el extremo de menos responsabilidad y reconocimiento para el alumnado, i.e. acatar y respetar lo decidido por otros, que sería la que haría referencia más directa a las falacias de la participación señaladas por Santos Guerra (2007). Si bien en las concepciones del alumnado parece haber calado el significado de participación que se defiende desde un modelo de justicia social y de inclusión, se hace necesario comprobar si esas representaciones tienen su reflejo en su comportamiento real en los centros o si por el contrario nos encontramos con alumnos con baja implicación personal en la acción real.

En relación al segundo objetivo, les preguntábamos cómo estimaban ellos la participación del alumnado en sus centros y en sus aulas, y quién tenía la iniciativa de la misma. Encontramos que los alumnos consideran que su participación es alta o media, lo cual unido a la valoración que comentábamos anteriormente de la participación como requisito necesario para el buen funcionamiento de los centros, hace pensar en una buena satisfacción por su parte del rol que desempeñan en los centros. No obstante, al analizar la iniciativa nos encontramos con que la mitad de los estudiantes hablan de una participación reactiva, siendo el profesorado el que toma la iniciativa, y apenas reconociéndose una participación de carácter proactivo por parte del alumnado. Este carácter reactivo podría estar encerrando también otra de las falacias de la participación, la del juego democrático truncado ya que los alumnos y alumnas parecerían estar planteando una actitud sumisa y participando solo cuándo y dónde los docentes deciden (Santos Guerra, 2007) alejándose así de una situación ideal de poder compartido (Hart, 1992). Parece necesario profundizar en este aspecto para potenciar la participación real 
del alumnado y para poder plantear ámbitos en los que los estudiantes deseen participar por iniciativa propia. De la misma manera, parece necesario formar al profesorado de manera que sepa cómo dar voz y acción al alumnado en el proceso educativo entendiendo que es una práctica positiva y necesaria también de su quehacer docente (Saiz-Linares, Ceballos-López y Susinos-Rada, 2019)

Por último, nos gustaría resaltar algunas diferencias de género que hemos encontrado en el concepto de participación. En la línea de otros estudios que encontraban que las chicas tenían más confianza en la eficacia de la participación en la escuela (Flanagan et al., 1998), también hemos encontrado que son ellas quienes señalan más la necesidad de que el alumnado se muestre participativo para lograr que los centros funcionen de manera adecuada. De la misma manera, son ellas las que dan más peso al carácter voluntario de la participación, en línea con el modelo de derechos recogido por otros autores (Nussbaum, 2012; Save the Children, 2005). Dado que el enfoque de justicia social sería acorde con una ética de cuidado mutuo, y teniendo en cuenta las diferencias de género manifestadas, parece necesario valorar a las chicas como una palanca de cambio dentro de los centros escolares para hacerlos más inclusivos, sin dejar de lado el objetivo de conseguir que también los chicos sean parte activa de las decisiones protagonizadas por el alumnado.

\section{Referencias}

Biesta, G. (2016). Democracia, ciudadanía y educación: de la socialización a la subjetivación. Foro de Educación, 20, 21-34. https://doi.org/10.14516/fde.2016.014.020.003

Bisquerra, R. (2008). La educación para la ciudadanía y la convivencia. El enfoque de la educación emocional. Madrid: Wolters Kluwer.

Black- Hawkins, K., Florian, L. y Rouse, M. (2007). Achievement and inclusion in schools. Londres: Routledge.

Booth, T. (2006). Manteniendo el futuro con vida; convirtiendo los valores de la inclusión en acciones. En M. A. Verdugo y F. B. Jordán de Urríes (Coords.), Rompiendo inercias. Claves para avanzar. VI Jornadas Científicas de Investigación sobre personas con Discapacidad (pp. 211 217). Salamanca: Amarú.

Ceballos-López, N. y Saiz-Linares, A. (2019) Promoviendo la participación del alumnado en la escuela. Análisis de materiales y guías internacionales para docentes que desarrollan experiencias de voz del alumnado. Profesorado. Revista de Currículum y Formación del Profesorado, 23(1), 329-350. https://doi.org/10.30827/profesorado.v23i1.9157

Ceballos-López, N., Susinos-Rada, T. y García-Lastra, M. (2018). Espacios para jugar, para aprender. Espacios para relacionarse. Una experiencia de voz del alumnado en la escuela infantil (0-3 años). Estudios Pedagógicos, 44(3), 117-135. https://doi.org/10.4067/So71807052018000300117

Clark, A. (2010). Transforming children's spaces: children's and adults' participation in designing learning environments. Londres: Routledge. https://doi.org/10.4324/9780203857588

Cochran-Smith, M. (2010). Toward a theory of teacher education for social justice. En M. Fullan, A. Hargreaves, D. Hopkins y A. Lieberman (Eds.), Second international handbook of educational change (pp. 445-467). Dordrecht: Springer. https://doi.org/10.1007/978-90481-2660-6_27

Coiduras, J. L., Balsells, M. A., Alsinet, C., Urrea, A., Guadix, I. y Belmonte, O. (2016). La participación del alumnado en la vida del centro: Una aproximación desde la comunidad 
educativa. Revista Complutense de Educación, 27(2), 437-456

https://doi.org/10.5209/rev_RCED.2016.v27.n2.46353

Connell, R.W. (1993). Schools and social justice. Filadelfia, PE: Temple University Press.

Cook-Sather, A. (2014). The trajectory of student voice in educational research. New Zeland Journal of Educational Studies, 49(2), 131-148.

Covell, K., Howe, B. y McNeil, J. (2008). If there's a rat, don't leave it. Young children's understanding of their citizenship rights and responsibilities. Cambridge Journal of Education, 38(3), 32 1-339. https://doi.org/10.1080/03057640802286889

Cowie, H., Naylor, P., Talamelli, L., Chauhan, P. y Smith, P. K. (2002). Knowledge, use of and attitudes towards peer support: A 2-year follow-up to the prince's trust survey. Journal of Adolescence, 25, 453-467. https://doi.org/10.1006/jado.2002.0498

Cowie, H. y Wallace, P. (2000). Peer support in action: from bystanding to standing by. Londres: SAGE.

Del Barrio, C., Martín, E., Almeida, A. y Barrios, A. (2003). Del maltrato y otros conceptos relacionados con la agresión entre escolares y su estudio psicológico. Infancia \& Aprendizaje 26(1), 9- 24. https://doi.org/10.1174/02103700360536400

Del Barrio, C, Barrios, A., Granizo, L., van der Meulen, K., Andrés, S. y Gutiérrez, H. (2011). Contribuyendo al bienestar emocional de los compañeros: Evaluación del programa compañeros ayudantes en un instituto madrileño. European Journal of Education and Psychology, 4, 5-17. https://doi.org/10.30552/ejep.v4i 1.62

Delval, J. (2001). Descubrir el pensamiento de los niños. Introducción a la práctica del método clínico. Barcelona: Paidós.

Echeita, G. (2008). Inclusión y exclusión educativa. "Voz y quebranto" REICE. Revista Iberoamericana sobre Calidad, Eficacia y Cambio en Educación, 6(2), 9-18.

Echeita, G. (2014). Educación para la inclusión o educación sin exclusiones. Madrid: Narcea.

Edwards, D. y Mullis, F. (2003). Classroom meetings: Encouraging a climate of cooperation. Professional School Counseling, 7(1), 20-28.

Field, A. (2005). Discovering statistics using SPSS. Londres: SAGE.

Flanagan, C., Bowes, J., Johnson, B., Csapo, B. y Sheblanova, E. (1998). Ties that bind: Correlates of adolescents' civic commitments in seven countries. Journal of Social Issues, 54, 457-475. https://doi.org/10.1111/j.1540-4560.1998.tbo1230.x

Flutter, J. (2006). This place could help you learn': Student participation in creating better school environments. Educational Review, https://doi.org/10.1080/00131910600584116

Fraser, N. (2008). La justicia social en la era de la política de identidad: Redistribución, reconocimiento y participación. Revista de Trabajo, 4(6), 83-99.

García-Pérez, D. y Montero, I. (2014). Organización y fomento de la participación del alumnado en educación primaria. Un estudio cualitativo de casos. Multidisciplinary Journal of Educational Research, 4(2), 211-238.

García-Valcárcel, A. y Basilotta, V. (2017). Aprendizaje basado en proyectos (ABP): Evaluación desde la perspectiva de alumnos de educación primaria. Revista de Investigación Educativa, 35(1), 113-131. https://doi.org/10.6018/rie.35.1.246811

Granizo, L. (2011). El papel de la participación del alumnado en los institutos de educación secundaria. (Tesis doctoral). Universidad Autónoma de Madrid. 
Granizo, L. (2019). ¿Para qué venir a la escuela? La visión del alumnado. En M. C. Pérez-Fuentes, M. M. Molero, J. J. Gázquez, A. Martos, A. B. Barragán, M. M. Simón y M. Sisto (Comps.), Investigación en el ámbito escolar: Un acercamiento multidimensional a las variables psicológicas y educativas (pp. 205-210). Almería: ASUNIVEP.

Granizo, L., Naylor, P. y del Barrio, C. (2006). Análisis de las relaciones sociales de los alumnos con síndrome de asperger en las escuelas integradas de secundaria: Un estudio de casos. Revista de Psicodidáctica, 1 1(2), 281-292.

Hart, D., Atkins, R. y Donelly, T. M. (2006). Community service and moral development. En M. Killen y J. Smetana (Eds.), Handbook of moral development (pp. 93-115). Trenton, NJ: LEA.

Hart, R. (1992). Children's participation: From tokenism to citizenship. Florencia: UNICEF.

Haste, H. (2005). My voice, my vote, my community. Croydon: Nestlé Trust.

Kim, C. Y. (2015). Qhy research 'by' children? Rethinking the assumptions underlying the

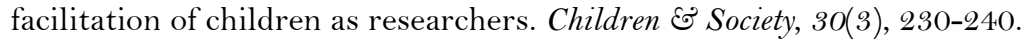
https://doi.org/10.1111/chso.12133

López, F. (2008). Necesidades en la infancia y en la adolescencia. Respuesta familiar, escolar y social. Madrid: Pirámide.

Martínez, J. B. y Aróstegui, J. L. (2001). La participación democrática del alumnado en los centros de educación secundaria. Revista de Educación, 36, 277-295

Mitra, D. (2018). Student voice in secondary schools: The possibility for deeper change. Journal of Educational Administration, 56(5), 473-487. https://doi.org/10.1108/JEA-01-2018-0007

Montero, I. y León, O. (2007). A guide for naming research studies in psychology. International Journal of Clinical and Health Psychology, 7(3), 847-862.

Murillo, F. J. y Hernández-Castilla, R. (2011). Hacia un concepto de justicia social. REICE. Revista Iberoamericana sobre Calidad, Eficacia y Cambio en Educación 9(4), 8-23.

Nussbaum, M. C. (2012). Crear capacidades. Propuestas para el desarrollo humano. Barcelona: Paidós.

Powell, M. A., Graham, A., Fitzgerald, R., Thomas, N. y White, N. E. (2018) Wellbeing in schools: What do student tell us? The Australian Educational Researcher, 45(4), 515-531. https://doi.org/10.1007/s13384-018-0273-Z

Power, F. C., Higgings, A. y Kohlberg, L. (1989). La educación moral según Lawrence Kohlberg. Barcelona: Gedisa. 2008.

Print, M. (2007). Citizenship education and youth participation in democracy. British Journal of Educational Studies, 55(3), 325-345. https://doi.org/10.1111/j.1467-8527.2007.00382.x

Pujolás, P. (2012). Aulas inclusivas y aprendizaje cooperativo. Educación Siglo XXI, 30(1), 89-112.

Quinn, S. y Owen, S. (2016). Digging deeper: Understanding the power of student voice. Australian Journal of Education, 60(1), 60-72. https://doi.org/10.1177/0004944115626402

Rodríguez, M., Kohen, R., Delval, J. y Messina, C. (2016). From democratic school to civic and political participation. Cultura y Educación 28, 99-129.

https://doi.org/10.1080/11356405.2015.1120449

Rudduck, J. y Flutter, J. (2003). Cómo mejorar tu centro escolar dando la voz al alumnado. Madrid: Morata. 2007.

Saiz-Linares, A., Ceballos-López, N. y Susinos-Rada, T. (2019). Voz del alumnado y mejora docente. Una investigación en centros educativos de Cantabria. Revista Complutense de Educación, 30(3), 713-728. https://doi.org/10.5209/rced.58883 
Santos Guerra, M. A. (2007). Arte y parte. Desarrollar la democracia en la escuela. Sevilla: Homo Sapiens Ediciones.

Save the Children. (2005). Estándares para la participación de la niñez. Londres: Alianza Internacional de Save the Children.

Serrano, D., Soto, Y., Arcos, E. y Jarquín, N. E. (2018). La participación infantil: La llave para la construcción de ambientes creativos en las escuelas. Revista Internacional de Educación para la Justicia Social, 7(2), 77-91. https://doi.org/10.15366/riejs2018.7.2.005

Simmons, C., Graham, A. y Thomas, N. (2015). Imagining an ideal school for wellbeing: Locating student voice. Journal of Educational Change, 16, 129-144. https://doi.org/10.1007/s 10833014-9239-8

Slee, R. (2010). The irregular school. Exclusion, schooling and inclusive education. Londres: Routledge. https://doi.org/10.4324/9780203831564

Susinos-Rada, T. y Ceballos-López, N. (2012). Voz del alumnado y presencia participativa en la vida escolar. Apuntes para una cartografía de la voz del alumnado en la mejora educativa. Revista de Educación, 359, 24-44.

Traver-Martí, J. A., Moliner, O. y Sales, A. (2019). Negociando el currículum: Aprendizaje-servicio en la escuela incluida. Alteridad, 14(2), 195-206. https://doi.org/10.17163/alt.v14n2.2019.04

UNESCO. (1996). La educación encierra un tesoro. Informe a la UNESCO de la Comisión Internacional sobre la educación para el siglo XXI, presidida por Jaques Delors. Madrid: Santillana. https://doi.org/10.22201/fcpys.2448492xe.1996.166.49503

UNESCO. (2001). The open files on inclusive education. París: UNESCO.

Uruñuela, P. (2018). La metodología del aprendizaje-servicio. Aprender mejorando el mundo. Madrid: Narcea.

Zabala, A. y Arnau, L. (2007). Cómo aprender y enseñar competencias: 11 ideas clave. Barcelona: Graó.

\section{Breve CV de los autores}

\section{Laura Granizo}

Profesora de la Facultad de Ciencias de la Salud y la Educación de la Universidad a Distancia de Madrid. Licenciada y Doctora en Psicología. Su investigación se centra en la mejora de la convivencia en los centros escolares, tanto por medio de medidas de centro como de aula, partiendo siempre del papel protagonista del alumnado. En esta línea, ha colaborado en la puesta en práctica de programas de ayuda entre iguales en centros de secundaria y ha participado en la adaptación del programa Equipar para Educadores para la mejora del clima moral de las aulas. Ha publicado en revistas nacionales e internacionales y ha llevado a cabo distintos procesos de formación con familias y docentes para mejorar la convivencia. Miembro del grupo de investigación INEXE: inclusión y exclusión educativa. ORCID ID: https://orcid.org/0000-0003-4937-9528. Email: laura.granizo@udima.es

\section{Kevin van der Meulen}

Profesor titular en la Facultad de Psicología de la Universidad Autónoma de Madrid y miembro del grupo de investigación INEXE. Imparte docencia en grado y posgrado relacionada con el desarrollo psicológico de niños y adolescentes desde 2001. Ha 
investigado el desarrollo psicológico de niños y adolescentes en diferentes ámbitos y la optimización de este desarrollo en la educación, como la naturaleza del acoso entre iguales, los efectos a largo plazo y la implantación de programas de mejora de la convivencia escolar centrados en la acción de los adolescentes. Su investigación actual sigue centrada tanto en las prácticas de mejora para la convivencia escolar como en el desarrollo de la identidad en varios aspectos en la adolescencia y adultez emergente. ORCID ID: https://orcid.org/0000-0002-0916-1551. Email: kevin.vandermeulen@uam.es

\section{Cristina del Barrio}

Catedrática de Psicología Evolutiva y de la Educación. Coordina el grupo de investigación INEXE. Sus líneas de investigación son: razonamiento social (identidad nacional, inmigración, guerra/paz, derechos); maltrato por abuso de poder (acoso, exclusión social): incidencia, representaciones en escolares de desarrollo típico y síndrome de Asperger; identidades diversas y desarrollo emocional; destreza manual. Entre otros proyectos (11 como IP/coIP), fue responsable del financiado por U. Europea sobre acoso escolar y su prevención y codirigió los dos Informes del Defensor del Pueblo. Ha publicado más de 60 capítulos de libro y artículos. Regularmente participa en congresos internacionales, e imparte conferencias (Ararteko-Defensor Pueblo; Cyberbullying ThinkTank, U. Arizona; Comparecencia en Comisión Educación del Congreso) y talleres para profesionales y ciudadanía (ayuntamientos, ONGs, etc.). ORCID ID: https://orcid.org/0000-0002-78292109. Email: cristina.delbarrio@uam.es 